\title{
Update of the American Society of Breast Surgeons Toolbox to address the lumpectomy reoperation epidemic
}

\author{
Maureen P. McEvoy ${ }^{1}$, Jeffrey Landercasper ${ }^{2}$, Himani R. Naik ${ }^{2}$, Sheldon Feldman ${ }^{1}$ \\ ${ }^{1}$ Montefiore Medical Center, Montefiore Einstein Center for Cancer Care, Bronx, NY, USA; ${ }^{2}$ Gundersen Health System, Norma J. Vinger Center \\ for Breast Cancer, La Crosse, WI, USA \\ Contributions: (I) Conception and design: MP McEvoy, J Landercasper, S Feldman; (II) Administrative support: None; (III) Provision of study materials \\ or patients: None; (IV) Collection and assembly of data: MP McEvoy, HR Naik, J Landercasper, S Feldman; (V) Data analysis and interpretation: MP \\ McEvoy, J Landercasper, S Feldman, HR Naik; (VI) Manuscript writing: All authors; (VII) Final approval of manuscript: All authors. \\ Correspondence to: Maureen P. McEvoy. 1521 Jarrett Place, Bronx, NY, USA. Email: mmcevoy@montefiore.org.
}

\begin{abstract}
In 2015, the American Society of Breast Surgeons (ASBrS) convened a multidisciplinary consensus conference, the Collaborative Attempt to Lower Lumpectomy Reoperation Rates (CALLER). The CALLER conference endorsed a "toolbox" of multiple processes of care for which there was evidence that they were associated with fewer reoperations. We present an update of the toolbox taking into consideration the latest advances in decreasing re excision rates. In this review, we performed a comprehensive review of the literature from 2015-2018 using search terms for each tool. The original ten tools were updated with the latest evidence from the literature and our strength of recommendation. We added an additional section looking at new tools and techniques that may provide more accurate intraoperative assessment of margins. The updates on the CALLER Toolbox for lumpectomy will help guide surgeons to various resources to aid in the removal of breast cancer, while being aware of cosmesis and decreasing re excision rates.
\end{abstract}

Keywords: Breast conservation; toolbox; re-operation rate; re-excision rate

Submitted Apr 16, 2018. Accepted for publication Nov 05, 2018.

doi: $10.21037 /$ gs.2018.11.03

View this article at: http://dx.doi.org/10.21037/gs.2018.11.03

\section{Introduction}

Reoperations after initial lumpectomy for breast cancer occur in about one in five patients and range from less than $10 \%$ to greater than $70 \%$ (1-6). The NSQIP database captures that reoperations after lumpectomy appear to be a high outlier compared to other organ sites (7). Addressing these high rates in 2015, the American Society of Breast Surgeons (ASBrS) convened a multidisciplinary consensus conference, the Collaborative Attempt to Lower Lumpectomy Reoperation Rates (CALLER). The CALLER conference endorsed a "toolbox" of multiple processes of care for which there was evidence that they were associated with fewer reoperations (8). The awareness of these high re excision rates have led to the descriptors of "over surgery", "epidemic", "over treatment" and "disutility" (9-12). This awareness also motivated the development of lumpectomy margin guidelines, enhanced preoperative imaging techniques, localization techniques and even novel tools at intra operative margin assessment. Our update on the toolbox takes into consideration the latest advances in decreasing re excision rates. The tools, references and strength of recommendation are described in Table 1.

We conducted a comprehensive review of the literature in PubMed from 2015 through 2018. We used different search terms specific for each topic as listed below.

\section{Update on toolbox}

Tool 1: preoperative diagnostic imaging should include full-field digital mammography and supplementary imaging to include ultrasound as needed

Background

There are multiple pre-operative imaging modalities, each 
Table 1 CALLER Toolbox update on references and strength of recommendation

\begin{tabular}{|c|c|c|c|c|}
\hline CALLER Toolbox tool & $\begin{array}{l}2015 \text { strength of } \\
\text { recommendation }\end{array}$ & $\begin{array}{l}2018 \text { strength of } \\
\text { recommendation }\end{array}$ & $\begin{array}{l}\text { Resource } \\
\text { intensiveness }\end{array}$ & $\begin{array}{l}\text { Updated } \\
\text { references }\end{array}$ \\
\hline $\begin{array}{l}\text { Preoperative diagnostic imaging should include full-field } \\
\text { digital mammography and supplementary imaging to } \\
\text { include ultrasound as needed }\end{array}$ & Strong-moderate & Similar & Moderate & $(13-21)$ \\
\hline $\begin{array}{l}\text { Minimally invasive breast biopsy (MIBB) for breast cancer } \\
\text { diagnosis }\end{array}$ & Strong & Similar & Low & - \\
\hline $\begin{array}{l}\text { For nonpalpable breast lesions, the use of radioactive } \\
\text { seeds, intraoperative US, or wire localization to direct } \\
\text { lesion excision is recommended }\end{array}$ & Strong & Similar & Moderate & $(32-42)$ \\
\hline Specimen radiograph with surgeon intraoperative review & Strong & Similar & Low & $(13,14,69-74)$ \\
\hline $\begin{array}{l}\text { Consider cavity shave margins in patients with T2 } \\
\text { or greater tumor size or } \mathrm{TI} \text { with extensive intraductal } \\
\text { carcinoma (EIC) }\end{array}$ & Strong-moderate & Strong & Low & $(75-77)$ \\
\hline $\begin{array}{l}\text { Intraoperative pathology assessment of lumpectomy } \\
\text { margins may help decrease re-excision when feasible }\end{array}$ & Strong-moderate & Strong & Highest & $(69,78-90)$ \\
\hline $\begin{array}{l}\text { Compliance with the SSO-ASTRO margin guideline to not } \\
\text { routinely re-operate for close margins with no tumor on ink } \\
\text { in patients with invasive cancer }\end{array}$ & Strong-moderate & Strong & Low & $\begin{array}{c}(4,5,8,11,91- \\
106)\end{array}$ \\
\hline
\end{tabular}

with their own advantages and disadvantages.

\section{Update}

The addition of tomosynthesis has helped detect breast cancers, especially in dense breast tissue. It is been shown to be more accurate in detecting tumors $(13,14)$.

Ultrasound (US) has emerged as one of the more accurate predictors of tumor size. Recent studies have compared mammogram, US and magnetic resonance imaging (MRI) to predict tumor size; concluding US was the most accurate (15). MRI overestimates tumor size. This was confirmed by Cortadellas et al. who found that US was the best predictor of tumor size when compared to the pathologic assessment of the surgical specimen (16).

The routine use of MRI is not standard of care. It is known to have an increased false positive rate and to be associated with the need for additional work up. Tseng et al. concluded that MRI provides greater accuracy for a third of patients undergoing preoperative mammogram and ultrasound (17). Some have recommended MRI in patients with dense breast tissue as part of the preoperative work up, however Elder et al. found no statistically significant difference in numbers of cancers detected or recurrence rates. This questions the routine use of MRI in breast cancer patients solely based on breast density (18). MRI has not been shown to improve disease free survival and overall survival (19). A population-based study in Canada showed preoperative MRI was associated with significant increase in ancillary investigations, wait time to surgery, mastectomies and contralateral prophylactic mastectomies (20).

Contrast enhance spectral mammography has shown promise in detecting tumor extent preoperatively, showing better size measurements with histopathologic size compared to full-field digital mammography and US (21).

\section{Conclusion}

Complete preoperative imaging is necessary; the type of modality is dependent on patient factors, comfort level of 
the surgeon and communication with the radiologist.

Search terms: complete diagnostic preoperative imaging breast surgery; preoperative imaging breast surgery.

* Total titles reviewed 48;

* Total abstracts reviewed 29;

* Total manuscripts reviewed 15;

* Total manuscripts used and referenced 9.

\section{Tool 2: minimally invasive breast biopsy (MIBB) for breast cancer diagnosis}

\section{Update}

There is no new evidence to refute that minimally invasive breast biopsy for breast cancer diagnosis is appropriate. It provides definitive diagnosis that allows for preoperative treatment planning in a multidisciplinary fashion.

Search terms: breast and lumpectomy and preoperative minimally invasive breast biopsy; breast conserving surgery and preoperative minimally invasive breast biopsy; core needle biopsy for breast cancer diagnosis and breast conserving surgery; core needle biopsy and open biopsy for breast cancer and breast conserving surgery.

* Total titles reviewed-no new articles from 2015 to 2018.

\section{Tool 3: multidisciplinary discussions to include radiology, patbology, surgery, and radiation and medical oncology}

\section{Background}

All participants in the ASBrS 2015 Consensus Conference to reduce reoperations recommended pre-operative multidisciplinary discussions to include representatives from the specialties of breast imaging, pathology, surgery, radiation oncology, medical oncology, genetics, and others. These recommendations were not based on level 1 evidence. They were based on personal, professional society, and other consensus conference recommendations, because to our knowledge, there had been no prospective randomized trials that stratified patients into a "non-multidisciplinary" arm.

\section{Update}

In an updated review of the literature, no studies with a high level of evidence to confirm the importance of pre-operative multidisciplinary planning to improve the outcome of re-excision rates were identified. Anecdotally, pre-operative multidisciplinary planning is increasing, resource-intensive and of value to patients. In an effort to quantify how often-multidisciplinary appointments were scheduled before surgery, Churilla et al. queried the SEER database (22). Among 88,865 patients, pre-operative multidisciplinary appointments were scheduled in only $2.9 \%$; only $14 \%$ of these had appointments with different providers the same day. These findings ought to motivate all stakeholders to improve the percentage of patients receiving preoperative multidisciplinary planning and to schedule these appointments according to the patients' requests if possible.

One part of pre-operative multidisciplinary planning that may impact reoperation rates is the decision whether the patient will receive neoadjuvant systemic therapy $(23,24)$. This provider-patient decision is discussed in more detail in the last section of this update.

In the absence of high level evidence informing surgeons of the association between multidisciplinary planning and reoperations, we refer readers to the organizations and their summary documents that describe the importance of multidisciplinary planning for many reasons, to include efforts to reduce reoperations (12,25-29).

In the strongest terms possible in a position statement, the European Union of Breast Cancer Specialists "demanded" that the countries of Europe provide sufficient funding so that all women have access to "multi-disciplinary and multi-professional" care (30).

\section{Out of scope}

Many other multidisciplinary tumor board and interdisciplinary topics of communication are emerging in the breast cancer literature. They are important for optimizing patient care and they may reduce reoperation rates by better collaboration and planning, but are out of scope for this update. One example can be found in the following reference (31).

Various other ideas to improve multidisciplinary communication include better interoperability of electronic medical and imaging records, and standardization and incorporation of a pre-operative tumor board summary into medical records with or without synoptic templates.

If complete multidisciplinary pre-operative patient planning is not possible for any reason, then communication between the breast surgeon and the breast radiologist should still occur to optimally localize the lesion to be excised, especially if it is non-palpable.

\section{Conclusion}

Pre-operative multidisciplinary planning-after a minimally invasive biopsy of the breast confirms breast canceris recommended for all patients prior to a planned breast 
conserving procedure.

Search terms: breast and multidisciplinary, breast tumor board, breast and interdisciplinary, breast lumpectomy and multidisciplinary, lumpectomy and multidisciplinary, breast and tumor board, breast and tumor conference.

* Total titles reviewed $>500$;

* Total abstracts reviewed 75;

* Total manuscripts reviewed 15;

* Manuscripts used and referenced 11.

Tool 4: for nonpalpable breast lesions, the use of radioactive seeds, intraoperative US, or wire localization to direct lesion excision is recommended

\section{Background}

A significant number of breast cancers are nonpalpable at the time of diagnosis (32). Image guided localization has been shown to decrease the positive margin rate and results in fewer re excisions (33). Currently the gold standard is wire-localization, however there are several new techniques of localization including radioactive seed, and non-radioactive device placement, each with its own advantages and disadvantages.

\section{Update}

A recent Cochrane review compared new forms of guided surgical intervention against wire-guided localization. They found there was no statistically significant difference between radio-guided occult lesion localization, radioactive seed localization versus wire localization (34). One major criticism of the wire localization technique is the workflow perspective, since the wire must be placed the day of surgery. This is a suboptimal patient experience (35). Hayes provided a review on localization techniques available today. She notes some of the advantages of non-wire devices include, avoids dislodgement or migrated wires, flexible surgery schedule for on time starting the operating room, improves surgical options of cosmetic approach, advance placement decouples the radiology-surgery schedule, and continuous intraoperative reorientation with target centering specimen (36).

By using bracketed technique larger resection areas are possible while preserving cosmesis. A retrospective review looked at DCIS with radioactive seed in single versus multiple, bracketed localization. They found that the rate of positive resection margin was $47.9 \%$ with single seed, compared to $29.3 \%$ with multiple seed localization. The reoperation rate was $39.6 \%$ versus $20.7 \%$ in single versus multiple seeds (37). Bracketing is possible with radioactive seeds, and has been shown to be superior with seeds than wire. A retrospective study showed a decrease in re excision rate with similar specimen volumes and lower rate of mastectomy for bracketed seed localization when compared with wire localization (38).

Cox et al. reported on the use of the SAVI SCOUT, concluding comparable re excision rates with other techniques, as well as improving the surgical approach allowing for optimal incision placement, improve operating room efficiencies and patient wait times (39). Radiolabeled seed localization has been shown to result in less pain, higher convenience rate with comparable excision volume and positive margin rates. SAVI allows for bracketing larger lesions, which can help define the borders of a more extensive area (40).

In a prospective randomized study Bloomquist et al. also showed higher patient satisfaction with radioactive seed localization compared to wire, resulting in less pain as well as higher convenience. As with similar studies they showed comparable excision volume and positive margin rate (41). Langhans et al. reported on a randomized control trail of radioactive seed localization versus wire guided for non-palpable cancers. They found no significant difference in the resection margins or duration of procedure. The main advantage was logistical (42).

\section{Conclusion}

There are several methods at localization of non-palpable cancers. The non-wire localizations are becoming more popular and have now been validated by randomized trails. They have been shown to facilitate patient and physician schedules. It also allows for more flexible incision placement. There is no difference in the positive margin rate. Surgeons should perform the modality they are most comfortable with, being aware of the various options.

Search terms: localization technique for breast conserving surgery, localization technique for breast lumpectomy, specimen imaging breast conserving surgery, specimen imaging breast surgery.

* Total titles reviewed 28;

* Total abstracts reviewed 20;

* Total manuscripts reviewed 16;

* Manuscripts used and referenced in text 11.

\section{Tool 5: oncoplastic techniques can reduce the need for reoperation in anatomically suitable patients}

\section{Background}

Driven by the desire to prevent ipsilateral breast deformity 
after breast conserving surgery and to improve symmetry with the contralateral breast, the use of oncoplastic techniques after lumpectomy is increasing (43-46). The focus here is to update information on the opportunity to potentially lower reoperations by receipt of oncoplastic lumpectomy compared to standard lumpectomy alone. Other oncoplastic areas of interest, such as selection of technique and cosmetic and oncologic outcomes, are out of scope for this update.

\section{Is oncoplastic lumpectomy associated with fewer reoperations for margin reasons?}

To our knowledge, there is no level 1 evidence that oncoplastic lumpectomy compared to simple lumpectomy results in lower reoperation rates. However, there are systematic reviews of the literature, comparative effectiveness studies and case series with lower levels of evidence that increasingly support the notion that receipt of oncoplastic surgery is associated with fewer reoperations (43-59).

By literature review, the unadjusted rates of reoperation with oncoplastic lumpectomy are lower than those reported recently in 3 different national patient registries. The mean lumpectomy reoperation rates reported in the National Cancer Data Base and the ASBrS patient registry in 2014 were $23.6 \%$ and $21.6 \%$ respectively $(3,4)$. In 156 National Health Service Trusts in England, 11,032 (20\%) of 55,297 patients underwent reoperation (2). In contrast, in a recent systematic review of patients undergoing oncoplastic breastconserving surgery in the years that overlapped with the national registry data above, De La Cruz et al. reported on 55 studies that evaluated 6,011 patients from 1998 to 2015 (49). In 49 of these studies that reported positive margin status and reoperations, the overall rates were $10.8 \%$ (range, $0-40 \%$ ) and $6 \%$ (range, $0-27 \%$ ) respectively (49).

In 2017, in a different systematic review, Campbell et al. identified 13 studies that compared reoperation rates in those patients undergoing oncoplastic lumpectomy versus those undergoing standard lumpectomy (48). Eight studies reported a benefit regarding margin status and reoperations; five failed to demonstrate benefits. Many of these studies were limited by lack of clarity of margin status and margin definitions. Some reported reoperation rates without margin data; others reported margin status without reoperation rates and others added a comparator control group of patients undergoing mastectomy. The largest study in the review cited above had an oncoplastic lumpectomy sample size of 1,177 patients (46). These patients were retrospectively compared to patients undergoing standard lumpectomy [3,559], mastectomy [3,263], and mastectomy plus reconstruction $[2,608]$. Reoperation rates were not reported but the patients undergoing oncoplastic lumpectomy had fewer positive/close margins (5.8\%) compared to standard lumpectomy $(8.3 \%, \mathrm{P}=0.04)$.

\section{Post-operative complications}

A recent consensus panel concluded that oncoplastic lumpectomy was associated with an increased risk of post-operative complications compared to standard lumpectomy (43). Post-operative complications after oncoplastic lumpectomy to include fat necrosis, skin necrosis, seroma, hematoma and surgical site infection occurred in $14.3 \%$ of patients in a systematic review of the literature (49). In other reviews, increased complication rates have also been reported $(48,51)$.

\section{Radiation after oncoplastic lumpectomy}

Breast tissue is re-arranged with most oncoplastic techniques. Thus, the radiation oncologist is challenged during their attempts to target the breast tissue adjacent to the patient's resected cancer. Such targeting is especially relevant for patients recommended to receive a boost dose of radiation and for patients wanting to be candidates for accelerated and/or partial breast radiation. To address these concerns, a group of radiation oncology experts systematically reviewed the literature (60). They recommended that surgeons consider the application of clips to the tumor bed. Pre-operative radiation oncology consultation was also recommended for patients wanting oncoplastic reconstruction in order to discuss radiation boost options, the "impact of surgical technique on fractionation scheme", and the likelihood that accelerated or other forms of partial breast radiation may not be possible (60).

\section{The future}

In 2017, in Basil Switzerland, the "First international consensus conference on standardization of oncoplastic breast conserving surgery" was convened. The delegates agreed that there is a need for prospective multicenter trials to "optimize patient selection and for standardized criteria to qualify and accredit oncoplastic training centers" (43).

\section{Conclusion}

In mostly unadjusted non-randomized retrospective reviews of patients undergoing initial breast conserving surgery, 
the reoperation rate is lower for margin reasons in those patients receiving oncoplastic lumpectomy compared to those that undergo lumpectomy without reconstruction. Oncoplastic procedures can also improve cosmetic outcomes and expand the population of patients eligible for breast conserving surgery.

The post-operative rates of surgical site infection and reoperations for reasons other than margins may be increased.

Pre-operative consultation with radiation oncology, prior to planned reconstruction that includes tissue rearrangement, and application of marking clips to the peri-tumoral tissue should be considered.

Nearly all systematic reviews of oncoplastic lumpectomy agree there is a need for prospective multicenter comparative effectiveness studies to further evaluate patient reported outcomes, cancer recurrence rates, complication rates and reoperation rates stratified by type of procedure.

Search terms: breast and oncoplastic; lumpectomy and oncoplastic; breast conserving and oncoplastic.

* Total titles reviewed 299;

* Total abstracts reviewed 66;

* Total manuscripts reviewed 35;

* Manuscripts used and referenced 21.

\section{Tool 6: specimen orientation of 3 or more margins}

\section{Background}

Specimen orientation denotes any process used in the operating room during lumpectomy to correctly annotate the specimen's in vivo orientation in 3 dimensions. Orientation can be accomplished by surgical clips, commercial markers, dyes, sutures and other techniques.

Specimen orientation alone does not reduce reoperation rates. Specimen orientation was included in the 2015 ASBrS Consensus Conference Toolbox because of its efficacy to target the correct site for a focused breast re-excision in patients needing reoperation for margin reasons. If specimen orientation is omitted and a patient requires subsequent re excision for positive margins, then entire cavity re excision is necessary. As a result, higher resection volumes and worse cosmetic outcomes would be anticipated, compared to a targeted re excision.

There are many different techniques for specimen orientation. To our knowledge, they have not been tested in level 1 randomized trials for their comparative effectiveness. Despite the absence of high level evidence as to which of many methods is best for orientation, the majority of the participants in the CALLER consensus conference recommended application of ink to at least 3 sides of the lumpectomy specimen. Other methods are acceptable. In at least one report, restricting orientation to only 2 sides limited the accuracy of identification of other sides (61). Some conference participants recommended that all sides of the lumpectomy be inked.

\section{Is specimen orientation a measure of quality?}

In 2013, the ASBrS used a modified Delphi process to rank the importance of more than 100 domains of breast surgical care. After hierarchical ranking, specimen orientation was one of nine processes of surgical care that were endorsed as a "quality measure" $(62,63)$.

\section{Update}

In this update, we found no new studies concluding that any specific orientation method was superior to others. However, recent publications have identified some pitfalls and solutions to various orientation issues. For example, Patel et al. recommended a solution to the problem of "losing" orientation if a single localization method such as clipping "fails" due to accidental clip dislodgement (64). They advocated for redundancy of localization methods by using a marking board, clips and sutures. In another report, McClatchy III et al. described use of an organic "molecular" dye instead of standard surgical inking in cases in which surgeons wanted to employ specialized intra-operative optical imaging devices for margin assessment (65). They reported that standard inking prevented optical margin interrogation. In a different report, Taylor et al. described a method to better discriminate between a radioactive seed and a marking clip that were initially indistinguishable on routine specimen imaging. They recommended a change in settings to widen the window on the portable Picture Archiving and Communication System (PACS) (66). Without this maneuver, they could not confirm that the target seed was removed with the specimen. Lastly, Brice et al. identified a specific type of "red ink", employed for specimen orientation that created a pattern of micro-calcifications that were suspicious for cancer on specimen imaging (67). Other ink colors were not associated with this pattern. If not recognized, unnecessary cavity shaves based on specimen imaging could occur.

Other specimen topics such as intra-operative imaging of the specimen and intraoperative margin assessment techniques are updated elsewhere in this review. 
How often do breast surgeons comply with recommendations to perform specimen orientation? High levels of compliance have been achieved by American and Canadian surgeons $(62,63,68)$. In a performance summary generated by 473 ASBrS member surgeons, specimen orientation occurred in $98.6 \%$ of 275,619 patients (range, 11-100\%) after lumpectomy for cancer $(62,63)$.

\section{Conclusion}

Specimen orientation by any technique that allows the breast pathologist to orient all sides of the specimen during margin assessment is essential. To determine the optimal method of orientation, future comparative effectiveness studies should be considered.

Search terms: breast specimen orientation, breast lumpectomy and orientation, breast specimen lumpectomy orientation, breast lumpectomy and ink, Breast AND Lumpectomy AND specimen orientation, Breast AND Specimen orientation and more margins, Specimen Orientation AND Marker OR ink OR Clip AND re excision AND re operation Specimen AND Orientation AND Marker OR Ink AND Breast cancer AND Lumpectomy. Breast AND Specimen AND Margin AND Orientation.

* Total titles reviewed 64;

* Total abstracts reviewed 24;

* Total manuscripts reviewed 12;

* Manuscripts used and referenced 8.

\section{Tool 7: specimen radiograph with surgeon intraoperative} review

\section{Background}

Intraoperative specimen radiograph review has improved with several modalities available. The surgeon should review the imaging in conjunction with a radiologist to potentially avoid re excision.

\section{Update}

Various intraoperative imaging methods exist including radiograph, ultrasound, microcomputed CT and MRI. St John et al. reported on a meta-analysis of 35 studies and found that frozen section and cytology had the greatest accuracy. They found there was not sufficient data on some of the newer imaging techniques to effectively make a fair comparison (69). Operating room radiograph provides immediate surgery feedback, and has been shown to not significantly impact operative time, the rate or number of additional intraoperative margins excised or the width of final pathological margins (70). Miller et al. confirmed the accuracy of intra operative specimen radiograph to standard specimen mammography with a randomized trial. They found significantly reduced interpretation time, 1 minute versus 9 minutes for intraoperative mammography compared to standard specimen mammography. In addition, there was $100 \%$ concordance for target and $93 \%$ concordance for margins among the specimens (71). The use of $3 \mathrm{D}$ versus $2 \mathrm{D}$ mammography can be more accurate and was shown to reduce the re excision rate in $2.2 \%$ of patients (13). In addition, breast tomosynthesis has been shown to more accurately detect breast cancer intraoperatively (14). This has been confirmed by Amer et al., showing accuracy of $40 \%$ versus $69 \%$ between full field digital mammography and digital breast tomosynthesis. Sensitivity was also significantly better at $62 \%$ versus $77 \%$ (72). The use of intra operative US is another modality for imaging specimens and margin status. It has been shown to have a $94 \%$ specificity in detecting the breast lesion as well as positive margin (73). Finally, another modality is microcomputed tomography. Tang et al. showed that micro-CT had the best correlation coefficient with pathologic tumor dimension, followed by MRI, US and mammography. The advantage is the ability to measure the tumor in $3 \mathrm{D}$ (74).

\section{Conclusion}

There are multiple modalities for intraoperative specimen review. This should be conducted with proper communication between the breast surgeon and radiologist.

Search terms: Specimen imaging breast conserving surgery, Intraoperative specimen imaging breast conserving surgery, Specimen imaging breast surgery, Intraoperative imaging breast surgery.

* Total titles reviewed 18 ;

* Total abstracts reviewed 16;

* Total manuscripts reviewed 11;

- Manuscripts used and referenced in text 8 .

\section{Tool 8: consider cavity shave margins in patients with T2 or greater tumor size or Ti with extensive intraductal carcinoma (EIC).}

\section{Background}

Cavity shaves for margins are becoming more common practice, however is not yet the standard of care. There is now evidence to show they decrease the positive margin and re excision rate. 


\section{Update}

Chagpar et al. conducted a randomized control trial of cavity shave margins, proving that this technique halved the rates of positive margin and re excision rates (75). Since that time there have been several retrospective studies and some randomized controlled trials demonstrating the effectiveness of this technique. It has not been shown to adversely affect cosmetic outcomes. Corsi $e t$ al. found that avoiding cavity shaved margins expose patients to 6.2 -fold higher risk of positive margin and 5.46-fold higher risk of reintervention (76). The cost effectiveness has been looked at, and there has been no increase in cost doing cavity shaves due to significantly fewer reoperations (77).

\section{Conclusion}

Cavity shave margins reduce the positive margin and re excision rate by half without adding significant time or cost. This is an effective, precise way of decreasing the re excision rate in breast conservation patients.

Search terms: cavity shave margins breast conserving surgery, cavity shave margins breast conserving surgery re excision, cavity shave margins breast conserving surgery reoperation, cavity shave margins, cavity shaved margins, cavity shaved margins breast conserving surgery.

* Total titles reviewed 14;

* Total abstracts reviewed 10;

* Total manuscripts reviewed 7;

* Manuscripts used and referenced in text 3 .

\section{Tool 9: intraoperative pathology assessment of lumpectomy margins may help decrease re-excision when feasible}

\section{Background}

In a 2012 systematic review of the literature, Esbona et al. identified 37 studies that measured reoperations after lumpectomy and compared those patients undergoing lumpectomy with immediate intraoperative margin assessment-either imprint cytology or frozen section analysis to those that underwent post-operative permanent histopathologic assessment (78). The final re excision rates after lumpectomy were $35 \%, 11 \%$, and $10 \%$ for postoperative assessment, imprint cytology and frozen section analysis respectively $(\mathrm{P}<0.001)$. In 2014, Boughey et al. compared the 30-day reoperation rates after lumpectomy for cancer between the National Surgical Quality Improvement Project (NSQIP) and the Mayo Clinic-Rochester data bases (89). Their purpose was to determine if there were differences between a national sample of institutions that had diverse pathologic practices and a single institution that utilized frozen section analysis. In NSQIP, the reoperation rate was $13.2 \%$. At the Mayo Clinic-Rochester, the rate was $3.6 \%(\mathrm{P}<0.001)$; on multivariate analysis, the odds ratio for the likelihood of a reoperation in facilities reporting in NSQIP compared to the reference institution that used frozen section was greater than $4(\mathrm{P}<0.0001)(79)$.

On the evidence of these and other publications, the 2014 ASBrS Consensus Conference concluded that immediate methods of intra-operative margin assessment were associated with fewer reoperations compared to postoperative margin assessment. On the other hand, it was also noted that some institutions already had low rates of reoperation without intra-operative assessment. Additionally, for many institutions wanting to change their practice to one of routine frozen section, it was predicted that there would be significant, perhaps even insurmountable financial and staffing barriers to building the infrastructure for such a program.

\section{Update}

Since the 2014 Consensus Conference, multiple investigators have reviewed the association between intraoperative pathologic specimen evaluation of the lumpectomy margin and reoperation rates $(69,80-88)$. In a meta-analysis of these techniques in 2017, St John et al. reviewed 838 unique studies to identify 35 with sufficient data to determine pooled sensitivity, specificity, and area under the receiver operating characteristic curve (69). The respective values per group were as follows: frozen section $=86 \%, 96 \%, 0.96$; cytology $=91 \%, 95 \%, 0.98$; intraoperative ultrasound $=59 \%, 81 \%, 0.78$; specimen radiography $=53 \%, 84 \%, 0.73$; and optical spectroscopy $=85 \%, 87 \%, 0.88$. The authors concluded that intraoperative frozen section and cytology had the greatest accuracy.

In a systematic review of the literature in 2017, Gray et al. identified 106 publications that were focused on intraoperative management of lumpectomy specimens (80). After disentangling those studies relevant to immediate intraoperative pathologic examination from those related to specimen imaging, cavity shaves and specimen imaging, they identified those studies that focused specifically on pathologic techniques. Collectively, the patient sample size for these exceeded 10,000 (80). Due to the heterogeneity of margin definitions and the outcomes measured in these studies, they did not combine data for a pooled or meta-analysis. Upon completion of their review, the authors 
concluded that frozen section analysis and imprint cytology were associated with lower rates of positive margins. Using standard definitions for level of evidence and strength of their recommendation, the authors categorized frozen section and imprint cytology as $2 \mathrm{~B}$ and $2 \mathrm{C}$ respectively.

In 2018, 3 breast surgeons authored chapters in textbooks that also reviewed the literature on margin assessment to include intra-operative pathologic assessment techniques $(81,87,88)$. In each of these reviews, there was overlap of the publications already cited above. Notwithstanding this overlap, 2 of these 3 authors qualitatively concluded that the receipt of frozen section analysis and imprint cytology during lumpectomy were associated fewer reoperations $(81,88)$. The third author cited similar studies but emphasized the "shortcomings" of immediate intraoperative pathologic maneuvers to include the dual burdens of extra time-in-the$\mathrm{OR}$ and cost, as well as surface sampling errors, loss of tissue for biomarker evaluation and the inability of cytology to distinguish between invasive and in-situ cancer (87).

Patients with ductal carcinoma in situ (DCIS) without invasive cancer were not included in most of the aforementioned studies. In 2016, Decker et al. reviewed the role of intraoperative frozen section and touch prep in 8,259 DCIS patients captured in the Surveillance, Epidemiology, and End Results-Medicare data base. In this report, intraoperative assessment was not associated with any reduction in reoperations (adjusted OR 1.07; 95\% CI, 0.95-1.21; $\mathrm{P}=0.293)(85)$.

To our knowledge, no randomized trials have been conducted to compare intra-operative to postoperative pathologic assessments of lumpectomy specimen margins for the outcomes of reoperation rates, direct costs (pathologist, technician, equipment), and indirect costs (training, time-in-OR, scheduling difficulty). Additionally, it is unclear whether the excellent results achieved by intraoperative frozen section in higher volume institutions with experienced breast pathologists would be reproducible in lower volume non-specialized centers.

\section{Cost effectiveness of frozen section}

Boughey et al. built an economic model to assess annual costs associated with lumpectomy with and without frozen section. In the model, reoperation rates without frozen section were estimated from the literature at $15-50 \%$. Reoperation rates with frozen section were based on a measured institutional performance of $3 \%$ (89). The patient population in the model was based on Medicare charge data; costs from other public and private payers were estimated. Payer and provider costs were then calculated for differing rates of diffusion of frozen section into national practice. Limitations within this model were acknowledged. Indirect costs to providers and facilities were not included. Furthermore, total direct and indirect costs to the patient were not in the model because copays, time away from work and other costs were not known. A Monte Carlo analysis with 10,000 simulations was conducted. The simulations were of differing rates of diffusion of incorporation of frozen section into the United States and a facility's baseline reoperation rate. The authors concluded that the incorporation of frozen section into practice offered cost savings to payers and was neutral to a slight cost saving to providers. For two specific scenarios, the estimated potential annual cost savings were $\$ 18.2$ million to payers and $\$ 0.4$ million to providers when frozen section margin analysis was used in $20 \%$ of national breast lumpectomies; savings increased to $\$ 90.9$ million and $\$ 1.8$ million if $100 \%$ of facilities adopted frozen section (89).

Arguing against the potential advantages to greater diffusion of frozen section techniques as described above, others have emphasized that the disruptions associated with its adoption, such as those associated with pathology staffing, work-flow, extra time to perform cases-coinciding with less availability of operating room access-make routine frozen section "untenable" (90). Others have emphasized the technical challenges of freezing adipose tissue, margin sampling errors, slow reporting times and logistics $(87,88)$.

\section{Conclusion}

Reoperation rates after initial lumpectomy for cancer are significantly lower in those facilities utilizing routine intraoperative frozen section or imprint cytology for margin assessment compared to national and international average rates of reoperation.

The cost-effectiveness and reproducibility of the accuracy of frozen section techniques outside of centers that have already verified excellent results is unknown. For institutions wanting to adopt these techniques, we recommend they audit their results.

Search terns: breast lumpectomy frozen section, breast lumpectomy frozen section systematic review, breast lumpectomy touch prep, breast lumpectomy imprint cytology, breast lumpectomy intraoperative.

* Total titles reviewed 220;

* Total abstracts reviewed 64;

* Total manuscripts reviewed 27;

* Manuscripts used and referenced 12. 
Tool 10: compliance with the SSO-ASTRO margin guideline to not routinely re-operate for close margins with no tumor on ink in patients with invasive cancer

\section{Background}

In 2014, the SSO-ASTRO Consensus Conference issued a Margin Statement recommending that surgeons omit re-excision lumpectomy in patients with "close" but "ink-negative" margins after an initial lumpectomy. This recommendation was based on a meta-analysis of patients with invasive breast cancer that clarified the association between lumpectomy margin status and margin width with later in-breast tumor recurrence $(91,92)$. Soon thereafter, the ASBrS and many others endorsed the SSO-ASTRO Margin Statement $(8,93)$.

In 2016, a second SSO-ASTRO Margin Statement that focused on patients with ductal carcinoma in situ was published (94). The meta-analysis that underpinned this statement found that margin widths greater than $2 \mathrm{~mm}$ were not associated with any further reduction in cancer recurrence compared to $2 \mathrm{~mm}$ (95). Since the publication of both SSO-ASTRO Consensus Statements, multiple investigators have queried patient registries to determine whether reoperation rates decreased.

Based on empirical evidence, there was much hope that avoiding re-excision in patients with close, but ink-negative margins would reduce reoperation rates in patients with invasive cancer. For example, in 2013, 328 surgeons entered information into the ASBrS Mastery patient registry on 6,725 patients undergoing initial lumpectomy for cancer. Of those patients who underwent re-excision, the primary reason for the re-excision was a close but ink-negative margin in $40 \%$ (4).

Evidence that reoperations decreased after the margin statement

Multiple investigators concluded that reoperations decreased in comparison of rates before and after the 2014 Margin Statement (5,11,96-99). Two studies utilized national registries. Among 252 surgeons entering data into the ASBrS Mastery registry on 26,102 patients undergoing initial lumpectomy, the reoperation rates decreased from $20.2 \%$ to $16.5 \%(\mathrm{P}<0.001)$; there was a $13.8 \%$ decrease in reoperations performed for the reason of close margins (5). In a population-based cohort sample from the Surveillance, Epidemiology, and End Results registry, Morrow et al. reported a $16 \%(\mathrm{P}<0.001)$ absolute reduction in reoperation rates, accompanied by a breast conserving therapy rate that improved from $52 \%$ to $65 \%$ (11).

Most single institution studies of reoperation rates before and after the 2014 Margin Statement have similar conclusions (96-98). The University of Louisville noted a decrease in reoperation rate from $37 \%$ to $9 \%, \mathrm{P}<0.001$ ( $\mathrm{N}=237$ patients) (96). At Cedars-Sinai in Los Angeles, Chung et al. reported a decrease from $19 \%$ to $13 \%, \mathrm{P}=0.03$ ( $\mathrm{N}=846$ patients). At the Memorial Sloan Kettering Cancer Center, Rosenberger et al. reported a decrease from $21.4 \%$ to $15.1 \%, \mathrm{P}=0.006$ ( $\mathrm{N}=1,205$ patients), and at the Carolinas HealthCare System, Patten $e t$ al. reported a decrease from $20.4 \%$ to $16.3 \%, \mathrm{P}=0.104$ ( $\mathrm{N}=954)(97-99)$.

\section{Prediction of improvement in reoperation rates and costs of care based on retrospective application of margin statements}

Multiple studies have estimated the anticipated improvement in reoperation rates if there was compliance with the Margins Statements (100-103). In the United Kingdom and Ireland, Tang et al. reported data on reoperations and margin status from 79 breast units during 2016 (100). The overall mean reoperation rate was $17.2 \%$ (range, $0-41 \%$ ). After retrospective review of margins, the authors predicted a reduction in their overall rate to $15 \%$ if all units had consistently applied the SSO-ASTRO Statement. Yu et al. applied the SSO Guideline retrospectively to patients treated at a single institution and estimated a $5.6 \%$ decrease in reoperation rates and 2,360 \$ per patient cost savings (101). In British Columbia, Canada, investigators estimated their reoperation rate for invasive cancer would decrease from $25 \%$ to $16 \%$ by omitting resection in patients with close margins and Merrill et al., at Massachusetts General Hospital, estimated that applying the 2014 SSO Guidelines to a 2004-2006 patient cohort would have reduced reoperations by $50 \%$ $(102,103)$.

The benefits of decreasing reoperation rates by adoption of the margin statement include reducing the cost burdens for patients and payers (104).

\section{No improvement}

At the University of Colorado School of Medicine, Gladden et al. noted re-excision rates of $11.9 \%$ before and $10.9 \%$ $(\mathrm{P}=0.65)$ after the 2014 Consensus Statement (105).

\section{College of American Pathology (CAP) protocols for margin assessment}

In 2018, Guidi et al. surveyed more than 700 pathologists 
enrolled in a breast pathology performance improvement project (106). They reported that there were opportunities to improve compliance with CAP protocols for lumpectomy specimens. Twenty-three percent of 791 respondents performed en face instead of perpendicular margins and $12 \%$ did not ink separate cavity shave margins; both actions might preclude compliance. Thus it behooves surgeons to discuss margin processing techniques with their pathologists.

\section{Conclusion}

There is strong evidence that compliance with the SSOASTRO Margin Guidelines will reduce reoperation rates.

Search terms: margin statement and breast; SSO margin statement and Morrow; SSO margin statement and reoperations; SSO margin statement and re excision; SSO margin statement and lumpectomy; SSO margin statement and trends; breast and reoperations; and all the previous replacing "statement" with "guideline".

* Total titles reviewed 189;

* Total abstracts reviewed 42;

* Total manuscripts reviewed 30;

* Manuscripts used and referenced 19.

\section{Are there other opportunities for reducing reoperations after lumpectomy that were not endorsed by the ASBrS Consensus Conference in 2015?}

\section{Background}

The participants in the 2015 consensus conference on reoperations were asked to nominate any processes of care or adjuncts that had the potential to reduce reoperations and improve cosmetic outcomes. The final consensus statement endorsed eleven. Other processes and adjuncts were considered, such as margin devices, but not endorsed. The following discussion provides an update of margin devices.

In addition, two other processes of care are discussed for which there is some evidence of their efficacy to reduce reoperations. The processes of care discussed below have not been endorsed by the ASBrS; they represent the authors' perspective only.

\section{Margin devices}

There has been encouraging progress in the development of intraoperative devices to aid in the assessment of lumpectomy margins. The goal is to rapidly interrogate the lumpectomy specimen as well as the lumpectomy cavity to evaluate for residual cancer. Ideally, technology that would allow conservative lumpectomy with sequential assessment of shave margins and the cavity are being optimized. This would be analogous to the MOHS method which is used to assess margins for cutaneous malignancies. Some of the emerging modalities have the added benefit of evaluating nodal disease. The MarginProbe (MP) has the largest body of data and has approval from the US Food and Drug Administration (FDA). It uses radiofrequency spectroscopy and has demonstrated a significant relative reduction (23-56\%) in reoperation rates in 2 randomized clinical trials $(107,108)$. Kupstas et al. in a retrospective review of 240 patients demonstrated a $50 \%$ reduction in re-excisions using the MP (109). Coble et al., compared 137 MP cases to 199 full cavity shave cases. The re excision rate was reduced by $57 \%(\mathrm{P}=0.026)$, from $15.1 \%$ to $6.6 \%$. The overall tissue volume removed was reduced by $32 \%(\mathrm{P}=0.0023)$, from 115 to $78 \mathrm{cc}(110)$. Sebastian et al. reported on the use of MP in 165 patients with a margin re-excision rate of $9.9 \%$ compared to $25.8 \%$ in their historical controls (111). There has been the clinical observation that the false positive rate for the MP is higher in the setting of dense breast tissue. Blohmer et al. reported a prospective clinical study of 150 patients. They report an overall reduction in re-excision rates of $14.6 \%$ compared to historical controls; which included some patients with DCIS, lobular histology and dense breast tissue (112). Another technology, the ClearEdge system evaluates the bioimpedance of breast tissue. Dixon et al. have reported sensitivity $84-87 \%$ for involved margins and specificity to confirm negative margins at $76-85 \%$ using this system (113).

Another approach uses optical coherence tomography (OCT) which enables real-time high-resolution imaging of tissue microstructure. In a translational study by EricksonBhatt et involving 35 patients, a handheld surgical OCT imaging probe was developed for in vivo use to assess margins both in the resection bed and on excised specimens for the microscopic presence of cancer. The ex vivo images were compared with standard postoperative histopathology to yield sensitivity of $91.7 \%$ and specificity of $92.1 \%$. This study demonstrates in vivo OCT imaging of the resection bed during lumpectomy has the potential for realtime microscopic image-guided surgery (114). Zysk et al. reported a multicenter, prospective, blinded study of 46 patients performed to test the feasibility of using handheld OCT probe with analysis the interpretation of 2,191 images from 229 shave margin specimens with promising results (115). Additional optical imaging modalities for intraoperative use are in development. Wang et al. have 
developed a Raman-encoded molecular imaging (REMI) technique in which targeted nanoparticles are topically applied on excised tissues to enable rapid visualization of a multiplexed panel of cell surface biomarkers at surgical margin surfaces (116). Thomas et al. describe automated 3-dimensional scanner using Raman spectroscopy (117). Mondal et al. have described the feasibility of an optical goggle augmented imaging and navigation system (GAINS) for near-infrared, fluorescence-guided surgery using indocyanine green (118). Unkart et al. describe the use of a novel, ratiometric activatable cell-penetrating peptide (AVB-620) in breast cancer surgery. Their study involving 26 patients demonstrated the safety and dose optimization with Intraoperative imaging with an optical camera of surgical lumpectomy and nodal specimens after infusion with AVB-620 allowed for real-time tumor detection based upon fluorescence (119). Clinical trials of this method are currently in progress. An emerging unique approach is being investigated by Takats and Leff investigating the role of rapid evaporative ionization mass spectrometry through online chemical analysis of electrosurgical aerosol toward real-time margin assessment as an "intelligent knife". Here as the lumpectomy is being performed the electrocautery smoke is collected and rapidly analyzed for cancer related by products which would indicate an involved surgical margin (90).

Another novel approach intraoperatively to reducing margin re-excision has been reported by Klimberg et al. using radiofrequency ablation (RFA). This approach treats a $1 \mathrm{~cm}$ zone of breast tissue with RFA around the periphery of the lumpectomy cavity during surgery (120). The concept is that if margins are close or involved that re-excision can be avoided since the adjacent tissue has been ablated by the RFA. This approach may also allow selected patients who undergo intraop RFA to forego adjuvant radiation. The ABLATE 1 trial accrued 100 patients. During the study mean follow-up period of $62 \pm 24$ months (68-month median follow-up) in patients not treated with XRT, there were 2 in-site tumor recurrences treated with aromatase inhibitor, 3 biopsy entrance site recurrences treated with excision and XRT to conserve the breast, and 2 recurrences elsewhere and 1 contralateral recurrence; all 3 treated with mastectomy. A larger multicenter trial (ABLATE 2) has favorable results (personal communication-Klimberg) which will be published in the near future.

Pursuit of a triple aim of breast conserving surgery (BCS): higher BCS rate, fewer reoperations and better cosmetic outcome with "extreme" oncoplastic lumpectomy and neoadjuvant chemotherapy.

The primary aim of oncoplastic lumpectomy is to improve the cosmetic outcome of the breast compared to standard lumpectomy. A potential secondary gainas described earlier-may be fewer reoperations. An international consensus conference and others have described an additional advantage to oncoplastic lumpectomy. Patients with larger or multifocal tumorsinitially deemed ineligible for breast conservation-can be offered larger volume oncoplastic resection techniques to extend the indications for breast conservation (43-45). Silverstein et al. have termed this "extreme" oncoplasty and have reported low reoperation rates even in patients with tumor extent exceeding $5 \mathrm{~cm}(44,45)$. In 2017, the "First international consensus conference on standardization of oncoplastic breast conserving surgery" concluded that oncoplastic techniques can "broaden" the indication for breast conserving surgery (BCS) towards larger tumors, potentially increasing the BCS rate.

Neoadjuvant chemotherapy (NAC), compared to postoperative adjuvant chemotherapy, may be able to achieve similar objectives (24). In the National Cancer Data Base (NCDB), patients with receipt of NAC, compared to those that did not, had a higher BCS rate (121). In a separate investigation of the NCDB, 71,627 stage I-III breast cancer patients were identified that received chemotherapy. In this cohort, the receipt of chemotherapy before instead of after surgery was associated with fewer reoperations after initial lumpectomy for breast cancer (24). After propensity score matching of patients and multivariate analysis, the relative odds of reduction in reoperation rates were almost halved [0.53 (0.49-0.57), $\mathrm{P}<0.001]$. The odds of reduction in reoperation rates were even higher in patients with triple negative and HER 2 overexpression status.

A recent systematic review of the literature also assessed reoperation rates (and other surgical outcomes) in patients with receipt of NAC compared to those that did not. In 26 publications ( $\mathrm{N}=15,489$ patients), Volders et al. identified mixed results insofar as the effectiveness of NAC to reduce reoperations after lumpectomy (23). This systematic review was limited by heterogeneity of performance by facility and publication dates. During these dates, adjuvant therapies were changing and there was wide variability of performance. Other limitations included non-uniform margin definitions, a wide range of reoperation rates between institutions and small sample sizes per facility.

Supporting the adoption of NAC and oncoplastic techniques to improve the care of selected patients 
undergoing breast conserving surgery, Broecker et al. recently reported using both in patients with higher stage cancers and reported acceptable cosmetic results, reoperation rates and cancer recurrence rates (122).

In 2017, the National Comprehensive Cancer Network and the St. Gallen International Expert Consensus Conference on the Primary Therapy of Early Breast Cancer 2017 have endorsed the option of neo-adjuvant systemic therapy to increase breast conservation rates $(25,29)$. The St. Gallen panel recommends NAC as "preferred" first treatment, instead of primary surgery, in patients with Stage II and III triple negative and HER 2 amplified cancers. The St. Gallen Conference also recommended that the breast excisional volume be limited to only the perceived residual tumor bed after NAC; a "de-escalation" of treatment that potentially could result in a better cosmetic outcome, regardless of regardless of receipt of an oncoplastic technique (25).

\section{Conclusion}

In eligible patients, oncoplastic lumpectomy, even in patients with large tumors, and/or neo-adjuvant chemotherapy, may help to achieve higher rates of breast conservation, lower rates of reoperation, and improvements in cosmetic outcomes.

\section{Conclusion and future directions}

The economic burden of margin re excision has been estimated to be about $\$ 18.8$ million per year (123). This impact of cost should help drive breast surgeons at using all modalities possible to decrease this cost. One method might be widespread use of frozen section margin analysis. Boughey et al. looked at the economic impact of this and found that if feasible, intraoperative frozen section analysis to guide the surgical management would be beneficial not only to patients but also financially for health care payers and providers (89).

The concept of over treatment in surgical management has been addressed by several authors. After the 2014 consensus statement endorsing a margin of "no ink on tumor" one study showed a decrease in surgery after initial lumpectomy by $16 \%$. This is encouraging to see positive results in a surgeon led initiative to address potential overtreatment (11). There is a focus to reduce re-excision rates. There are multiple factors, non-tumor related that lead to a positive margin including inadequate assessment of macroscopic disease extent at diagnosis, inaccurate impalpable disease localization and limited use of intraoperative specimen radiograph. Ultimately what constitutes an acceptable margin must be individualized within the context of the tumor size, biology, stage, and planned treatments (10).

The updates on the CALLER Toolbox for lumpectomy will help guide surgeons to various resources to aid in the removal of breast cancer, while being aware of cosmesis and decreasing re excision rates.

\section{Acknowledgements}

None.

\section{Footnote}

Conflicts of Interest: The authors have no conflicts of interest to declare.

\section{References}

1. McCahill LE, Single RM, Aiello Bowles EJ, et al. Variability in reexcision following breast conservation surgery. JAMA 2012;307:467-75.

2. Jeevan R, Cromwell DA, Trivella M, et al. Reoperation rates after breast conserving surgery for breast cancer among women in England: retrospective study of hospital episode statistics. BMJ 2012;345:e4505.

3. Wilke LG, Czechura T, Wang C, et al. Repeat surgery after breast conservation for the treatment of stage 0 to II breast carcinoma: a report from the National Cancer Data Base, 2004-2010. JAMA Surg 2014;149:1296-305.

4. Landercasper J, Whitacre E, Degnim AC, et al. Reasons for re-excision after lumpectomy for breast cancer: insight from the American Society of Breast Surgeons Mastery(SM) database. Ann Surg Oncol 2014;21:3185-91.

5. Schulman AM, Mirrielees JA, Leverson G, et al. Reexcision Surgery for Breast Cancer: An Analysis of the American Society of Breast Surgeons (ASBrS) Mastery(SM) Database Following the SSO-ASTRO "No Ink on Tumor" Guidelines. Ann Surg Oncol 2017;24:52-8.

6. Isaacs AJ, Gemignani ML, Pusic A, et al. Association of Breast Conservation Surgery for Cancer With 90Day Reoperation Rates in New York State. JAMA Surg 2016;151:648-55.

7. Eck DL, Koonce SL, Goldberg RF, et al. Breast surgery outcomes as quality measures according to the NSQIP database. Ann Surg Oncol 2012;19:3212-7. 
8. Landercasper J, Attai D, Atisha D, et al. Toolbox to Reduce Lumpectomy Reoperations and Improve Cosmetic Outcome in Breast Cancer Patients: The American Society of Breast Surgeons Consensus Conference. Ann Surg Oncol 2015;22:3174-83.

9. Cody HS 3rd, Van Zee KJ. Reexcision--The Other Breast Cancer Epidemic. N Engl J Med 2015;373:568-9.

10. MacNeill F, Karakatsanis A. Over surgery in breast cancer. Breast 2017;31:284-9.

11. Morrow M, Abrahamse P, Hofer TP, et al. Trends in Reoperation After Initial Lumpectomy for Breast Cancer: Addressing Overtreatment in Surgical Management. JAMA Oncol 2017;3:1352-7.

12. Ong WL, Schouwenburg MG, van Bommel ACM, et al. A Standard Set of Value-Based Patient-Centered Outcomes for Breast Cancer: The International Consortium for Health Outcomes Measurement (ICHOM) Initiative. JAMA Oncol 2017;3:677-85.

13. Chagpar AB, Butler M, Killelea BK, et al. Does threedimensional intraoperative specimen imaging reduce the need for re-excision in breast cancer patients? A prospective cohort study. Am J Surg 2015;210:886-90.

14. Urano M, Shiraki N, Kawai T, et al. Digital mammography versus digital breast tomosynthesis for detection of breast cancer in the intraoperative specimen during breastconserving surgery. Breast Cancer 2016;23:706-11.

15. Leddy R, Irshad A, Metcalfe A, et al. Comparative accuracy of preoperative tumor size assessment on mammography, sonography, and MRI: Is the accuracy affected by breast density or cancer subtype? J Clin Ultrasound 2016;44:17-25.

16. Cortadellas T, Argacha P, Acosta J, et al. Estimation of tumor size in breast cancer comparing clinical examination, mammography, ultrasound and MRI-correlation with the pathological analysis of the surgical specimen. Gland Surg 2017;6:330-5.

17. Tseng J, Kyrillos A, Liederbach E, et al. Clinical accuracy of preoperative breast MRI for breast cancer. J Surg Oncol 2017;115:924-31.

18. Elder EA, Ferlin A, Vallow LA, et al. The Influence of Breast Density on Preoperative MRI Findings and Outcome in Patients with a Known Diagnosis of Breast Cancer. Ann Surg Oncol 2017;24:2898-906.

19. Choi WJ, Cha JH, Kim HH, et al. Long-term Survival Outcomes of Primary Breast Cancer in Women With or Without Preoperative Magnetic Resonance Imaging: A Matched Cohort Study. Clin Oncol (R Coll Radiol) 2017;29:653-61.
20. Arnaout A, Catley C, Booth CM, et al. Use of Preoperative Magnetic Resonance Imaging for Breast Cancer:

A Canadian Population-Based Study. JAMA Oncol 2015;1:1238-50.

21. Patel BK, Garza SA, Eversman S, et al. Assessing tumor extent on contrast-enhanced spectral mammography versus full-field digital mammography and ultrasound. Clin Imaging 2017;46:78-84.

22. Churilla TM, Egleston BL, Murphy CT, et al. Patterns of multidisciplinary care in the management of nonmetastatic invasive breast cancer in the United States Medicare patient. Breast Cancer Res Treat 2016;160:153-62.

23. Volders JH, Negenborn VL, Spronk PE, et al. Breastconserving surgery following neoadjuvant therapy-a systematic review on surgical outcomes. Breast Cancer Res Treat 2018;168:1-12.

24. Landercasper J, Bennie B, Parsons BM, et al. Fewer Reoperations After Lumpectomy for Breast Cancer with Neoadjuvant Rather than Adjuvant Chemotherapy: A Report from the National Cancer Database. Ann Surg Oncol 2017;24:1507-15.

25. Curigliano G, Burstein HJ, E PW, et al. De-escalating and escalating treatments for early-stage breast cancer: the St. Gallen International Expert Consensus Conference on the Primary Therapy of Early Breast Cancer 2017. Ann Oncol 2017;28:1700-12.

26. Cardoso F, Costa A, Senkus E, et al. 3rd ESO-ESMO International Consensus Guidelines for Advanced Breast Cancer (ABC 3). Ann Oncol 2017;28:3111.

27. Silverstein MJ, Recht A, Lagios MD, et al. Special report: Consensus conference III. Image-detected breast cancer: state-of-the-art diagnosis and treatment. J Am Coll Surg 2009;209:504-20.

28. Standard 1.2 of The National Accreditation Program for Breast Centers Standards Manual: Multidisciplinary Breast Care Conference. 2018. Available online: https://www.facs. org/quality-programs/napbc/standards/2018standards/ chapter1/standard-1_2

29. Cancer NGfb. Available online: https://www.nccn.org/ professionals/physician_gls/pdf/breast.pdf

30. Cardoso F, Cataliotti L, Costa A, et al. European Breast Cancer Conference manifesto on breast centres/units. Eur J Cancer 2017;72:244-50.

31. Kuerer HM, Smith BD, Chavez-MacGregor M, et al. DCIS Margins and Breast Conservation: MD Anderson Cancer Center Multidisciplinary Practice Guidelines and Outcomes. J Cancer 2017;8:2653-62. 
32. Haakinson DJ, Stucky CC, Dueck AC, et al. A significant number of women present with palpable breast cancer even with a normal mammogram within 1 year. Am J Surg 2010;200:712-7; discussion 717-8.

33. Acosta JA, Greenlee JA, Gubler KD, et al. Surgical margins after needle-localization breast biopsy. Am J Surg 1995;170:643-5; discussion 645-6.

34. Chan R. Cochrane review summary for cancer nursing: drug therapy for the management of cancer-related fatigue. Cancer Nurs 2011;34:250-1.

35. Sharek D, Zuley ML, Zhang JY, et al. Radioactive seed localization versus wire localization for lumpectomies: a comparison of outcomes. AJR Am J Roentgenol 2015;204:872-7.

36. Hayes MK. Update on Preoperative Breast Localization. Radiol Clin North Am 2017;5 5:591-603.

37. Janssen NNY, van la Parra RFD, Loo CE, et al. Breast conserving surgery for extensive DCIS using multiple radioactive seeds. Eur J Surg Oncol 2018;44:67-73.

38. Da Silva M, Porembka J, Mokdad AA, et al. Bracketed radioactive seed localization vs bracketed wire-localization in breast surgery. Breast J 2018;24:161-6.

39. Cox CE, Russell S, Prowler V, et al. A Prospective, Single Arm, Multi-site, Clinical Evaluation of a Nonradioactive Surgical Guidance Technology for the Location of Nonpalpable Breast Lesions during Excision. Ann Surg Oncol 2016;23:3168-74.

40. Jadeja PH, Mango V, Patel S, et al. Utilization of multiple SAVI SCOUT surgical guidance system reflectors in the same breast: A single-institution feasibility study. Breast J 2018;24:531-4.

41. Bloomquist EV, Ajkay N, Patil S, et al. A Randomized Prospective Comparison of Patient-Assessed Satisfaction and Clinical Outcomes with Radioactive Seed Localization versus Wire Localization. Breast J 2016;22:151-7.

42. Langhans L, Tvedskov TF, Klausen TL, et al. Radioactive Seed Localization or Wire-guided Localization of Nonpalpable Invasive and In Situ Breast Cancer: A Randomized, Multicenter, Open-label Trial. Ann Surg 2017;266:29-35.

43. Weber WP, Soysal SD, El-Tamer M, et al. First international consensus conference on standardization of oncoplastic breast conserving surgery. Breast Cancer Res Treat 2017;165:139-49.

44. Silverstein MJ. Radical Mastectomy to Radical Conservation (Extreme Oncoplasty): A Revolutionary Change. J Am Coll Surg 2016;222:1-9.

45. Silverstein MJ, Savalia N, Khan S, et al. Extreme oncoplasty: breast conservation for patients who need mastectomy. Breast J 2015;21:52-9.

46. Carter SA, Lyons GR, Kuerer HM, et al. Operative and Oncologic Outcomes in 9861 Patients with Operable Breast Cancer: Single-Institution Analysis of Breast Conservation with Oncoplastic Reconstruction. Ann Surg Oncol 2016;23:3190-8.

47. Weber WP, Soysal SD, Zeindler J, et al. Current standards in oncoplastic breast conserving surgery. Breast 2017;34 Suppl 1:S78-81.

48. Campbell EJ, Romics L. Oncological safety and cosmetic outcomes in oncoplastic breast conservation surgery, a review of the best level of evidence literature. Breast Cancer (Dove Med Press) 2017;9:521-30.

49. De La Cruz L, Blankenship SA, Chatterjee A, et al. Outcomes After Oncoplastic Breast-Conserving Surgery in Breast Cancer Patients: A Systematic Literature Review. Ann Surg Oncol 2016;23:3247-58.

50. Losken A, Hart AM, Broecker JS, et al. Oncoplastic Breast Reduction Technique and Outcomes: An Evolution over 20 Years. Plast Reconstr Surg 2017;139:824e-33e.

51. Losken A, Hart AM, Chatterjee A. Updated Evidence on the Oncoplastic Approach to Breast Conservation Therapy. Plast Reconstr Surg 2017;140:14S-22S.

52. Papanikolaou IG. Oncoplastic breast-conserving surgery in breast cancer treatment Systematic review of the literature. Ann Ital Chir 2016;87:199-208.

53. Yiannakopoulou EC, Mathelin C. Oncoplastic breast conserving surgery and oncological outcome: Systematic review. Eur J Surg Oncol 2016;42:625-30.

54. Piper ML, Esserman LJ, Sbitany H, et al. Outcomes Following Oncoplastic Reduction Mammoplasty: A Systematic Review. Ann Plast Surg 2016;76 Suppl 3:S222-6.

55. De Lorenzi F, Hubner G, Rotmensz N, et al. Oncological results of oncoplastic breast-conserving surgery: Long term follow-up of a large series at a single institution: A matched-cohort analysis. Eur J Surg Oncol 2016;42:71-7.

56. Crown A, Wechter DG, Grumley JW. Oncoplastic Breast-Conserving Surgery Reduces Mastectomy and Postoperative Re-excision Rates. Ann Surg Oncol 2015;22:3363-8.

57. Hernanz F, Gonzalez-Noriega M, Sanchez S, et al. Oncoplastic breast conserving surgery with tailored needle-guided excision. Gland Surg 2017;6:698-705.

58. Challoner T, Skillman J, Wallis K, et al. Oncoplastic techniques: Attitudes and changing practice amongst breast and plastic surgeons in Great Britain. Breast 
2017;34:58-64.

59. Clough KB, Gouveia PF, Benyahi D, et al. Positive Margins After Oncoplastic Surgery for Breast Cancer. Ann Surg Oncol 2015;22:4247-53.

60. Yoon JJ, Green WR, Kim S, et al. Oncoplastic breast surgery in the setting of breast-conserving therapy: A systematic review. Adv Radiat Oncol 2016;1:205-15.

61. Molina MA, Snell S, Franceschi D, et al. Breast specimen orientation. Ann Surg Oncol 2009;16:285-8.

62. Landercasper J, Bailey L, Buras R, et al. The American Society of Breast Surgeons and Quality Payment Programs: Ranking, Defining, and Benchmarking More Than 1 Million Patient Quality Measure Encounters. Ann Surg Oncol 2017;24:3093-106.

63. Landercasper J, Fayanju OM, Bailey L, et al. Benchmarking the American Society of Breast Surgeon Member Performance for More Than a Million Quality Measure-Patient Encounters. Ann Surg Oncol 2018;25:501-11.

64. Patel J, Jenkins S. A technique for marking oncological breast tissue specimens. Ann Med Surg (Lond) 2016;7:7-8.

65. McClatchy DM 3rd, Krishnaswamy V, Kanick SC, et al. Molecular dyes used for surgical specimen margin orientation allow for intraoperative optical assessment during breast conserving surgery. J Biomed Opt 2015;20:040504.

66. Taylor D. What to look for on a breast specimen radiograph: lessons learnt. BMJ Case Rep 2015;2015:bcr2014206827.

67. Brice ME, Gossweiler M, Bennett L. Technical Considerations for Red Marking Ink Use When Interpreting Specimen Radiographs: Case Report. Radiology 2017;282:676-80.

68. Lovrics P, Hodgson N, O'Brien MA, et al. Results of a surgeon-directed quality improvement project on breast cancer surgery outcomes in South-Central Ontario. Ann Surg Oncol 2014;21:2181-7.

69. St John ER, Al-Khudairi R, Ashrafian H, et al. Diagnostic Accuracy of Intraoperative Techniques for Margin Assessment in Breast Cancer Surgery: A Meta-analysis. Ann Surg 2017;265:300-10.

70. Rhee D, Pockaj B, Wasif N, et al. Operative outcomes of conventional specimen radiography versus in-operating room specimen radiography in radioactive seed-localized segmental mastectomies. Am J Surg 2018;215:151-4.

71. Miller CL, Coopey SB, Rafferty E, et al. Comparison of intra-operative specimen mammography to standard specimen mammography for excision of non-palpable breast lesions: a randomized trial. Breast Cancer Res Treat 2016;155:513-9.

72. Amer HA, Schmitzberger F, Ingold-Heppner B, et al. Digital breast tomosynthesis versus full-field digital mammography-Which modality provides more accurate prediction of margin status in specimen radiography? Eur J Radiol 2017;93:258-64.

73. Moschetta $M$, Telegrafo $M$, Introna $T$, et al. Role of specimen US for predicting resection margin status in breast conserving therapy. G Chir 2015;36:201-4.

74. Tang R, Buckley JM, Fernandez L, et al. Microcomputed tomography (Micro-CT): a novel approach for intraoperative breast cancer specimen imaging. Breast Cancer Res Treat 2013;139:311-6.

75. Chagpar AB, Killelea BK, Tsangaris TN, et al. A Randomized, Controlled Trial of Cavity Shave Margins in Breast Cancer. N Engl J Med 2015;373:503-10.

76. Corsi F, Sorrentino L, Bonzini M, et al. Cavity Shaving Reduces Involved Margins and Reinterventions Without Increasing Costs in Breast-Conserving Surgery: A Propensity Score-Matched Study. Ann Surg Oncol 2017;24:1516-24.

77. Chagpar AB, Horowitz NR, Killelea BK, et al. Economic Impact of Routine Cavity Margins Versus Standard Partial Mastectomy in Breast Cancer Patients: Results of a Randomized Controlled Trial. Ann Surg 2017;265:39-44.

78. Esbona K, Li Z, Wilke LG. Intraoperative imprint cytology and frozen section pathology for margin assessment in breast conservation surgery: a systematic review. Ann Surg Oncol 2012;19:3236-45.

79. Osborn JB, Keeney GL, Jakub JW, et al. Cost-effectiveness analysis of routine frozen-section analysis of breast margins compared with reoperation for positive margins. Ann Surg Oncol 2011;18:3204-9.

80. Gray RJ, Pockaj BA, Garvey E, et al. Intraoperative Margin Management in Breast-Conserving Surgery: A Systematic Review of the Literature. Ann Surg Oncol 2018;25:18-27.

81. Reyna C, DeSnyder SM. Intraoperative Margin Assessment in Breast Cancer Management. Surg Oncol Clin N Am 2018;27:155-65.

82. Jorns JM, Daignault S, Sabel MS, et al. Frozen sections in patients undergoing breast conserving surgery at a single ambulatory surgical center: 5 year experience. Eur J Surg Oncol 2017;43:1273-81.

83. Ko S, Chun YK, Kang SS, et al. The Usefulness of Intraoperative Circumferential Frozen-Section Analysis of Lumpectomy Margins in Breast-Conserving Surgery. J 
Breast Cancer 2017;20:176-82.

84. Butler-Henderson K, Lee AH, Price RI, et al. Intraoperative assessment of margins in breast conserving therapy: a systematic review. Breast 2014;23:112-9.

85. Decker MR, Trentham-Dietz A, Loconte NK, et al. The Role of Intraoperative Pathologic Assessment in the Surgical Management of Ductal Carcinoma In Situ. Ann Surg Oncol 2016;23:2788-94.

86. Osako T, Nishimura R, Nishiyama Y, et al. Efficacy of intraoperative entire-circumferential frozen section analysis of lumpectomy margins during breastconserving surgery for breast cancer. Int J Clin Oncol 2015;20:1093-101.

87. Chagpar AB. Margins and Breast Cancer In: HowardMcNatt M, editor. Changing Paradigms in the Management of Breast Cancer, pp.59-69.

88. Grobmyer SR. Intraoperative evaluation of surgical margins in breast conserving surgery. In: Bland KI, Copeland EM 3rd, Klimberg VS, et al. The Breast: Comprehensive management of benign and malignant diseases. 2018:637-42.e3.

89. Boughey JC, Keeney GL, Radensky P, et al. Economic Implications of Widespread Expansion of Frozen Section Margin Analysis to Guide Surgical Resection in Women With Breast Cancer Undergoing Breast-Conserving Surgery. J Oncol Pract 2016;12:e413-22.

90. Leff DR, St John ER, Takats Z. Reducing the Margins of Error During Breast-Conserving Surgery: Disruptive Technologies or Traditional Disruptions? JAMA Surg 2017;152:517-8.

91. Moran MS, Schnitt SJ, Giuliano AE, et al. Society of Surgical Oncology-American Society for Radiation Oncology consensus guideline on margins for breastconserving surgery with whole-breast irradiation in stages I and II invasive breast cancer. J Clin Oncol 2014;32:1507-15.

92. Houssami N, Macaskill P, Marinovich ML, et al. The association of surgical margins and local recurrence in women with early-stage invasive breast cancer treated with breast-conserving therapy: a meta-analysis. Ann Surg Oncol 2014;21:717-30.

93. Landercasper J, Bailey L, Berry TS, et al. Measures of Appropriateness and Value for Breast Surgeons and Their Patients: The American Society of Breast Surgeons Choosing Wisely ((R)) Initiative. Ann Surg Oncol 2016;23:3112-8.

94. Morrow M, Van Zee KJ, Solin LJ, et al. Society of Surgical Oncology-American Society for Radiation Oncology-
American Society of Clinical Oncology Consensus Guideline on Margins for Breast-Conserving Surgery with Whole-Breast Irradiation in Ductal Carcinoma In Situ. Ann Surg Oncol 2016;23:3801-10.

95. Marinovich ML, Azizi L, Macaskill P, et al. The Association of Surgical Margins and Local Recurrence in Women with Ductal Carcinoma In Situ Treated with Breast-Conserving Therapy: A Meta-Analysis. Ann Surg Oncol 2016;23:3811-21.

96. Bhutiani N, Mercer MK, Bachman KC, et al. Evaluating the Effect of Margin Consensus Guideline Publication on Operative Patterns and Financial Impact of Breast Cancer Operation. J Am Coll Surg 2018;227:6-11.

97. Chung A, Gangi A, Amersi F, et al. Impact of Consensus Guidelines by the Society of Surgical Oncology and the American Society for Radiation Oncology on Margins for Breast-Conserving Surgery in Stages 1 and 2 Invasive Breast Cancer. Ann Surg Oncol 2015;22 Suppl 3:S422-7.

98. Rosenberger LH, Mamtani A, Fuzesi S, et al. Early Adoption of the SSO-ASTRO Consensus Guidelines on Margins for Breast-Conserving Surgery with WholeBreast Irradiation in Stage I and II Invasive Breast Cancer: Initial Experience from Memorial Sloan Kettering Cancer Center. Ann Surg Oncol 2016;23:3239-46.

99. Patten CR, Walsh K, Sarantou T, et al. Changes in margin re-excision rates: Experience incorporating the "no ink on tumor" guideline into practice. J Surg Oncol 2017;116:1040-5.

100. Tang SS, Kaptanis S, Haddow JB, et al. Current margin practice and effect on re-excision rates following the publication of the SSO-ASTRO consensus and ABS consensus guidelines: a national prospective study of 2858 women undergoing breast-conserving therapy in the UK and Ireland. Eur J Cancer 2017;84:315-24.

101.Yu J, Elmore LC, Cyr AE, et al. Cost Analysis of a Surgical Consensus Guideline in Breast-Conserving Surgery. J Am Coll Surg 2017;225:294-301.

102. Baliski CR, Pataky RE. Influence of the SSO/ASTRO Margin Reexcision Guidelines on Costs Associated with Breast-Conserving Surgery. Ann Surg Oncol 2017;24:632-7.

103. Merrill AL, Coopey SB, Tang R, et al. Implications of New Lumpectomy Margin Guidelines for Breast-Conserving Surgery: Changes in Reexcision Rates and Predicted Rates of Residual Tumor. Ann Surg Oncol 2016;23:729-34.

104. Greenup RA, Peppercorn J, Worni M, et al. Cost implications of the SSO-ASTRO consensus guideline on margins for breast-conserving surgery with whole breast 
irradiation in stage I and II invasive breast cancer. Ann Surg Oncol 2014;21:1512-4.

105. Heelan Gladden AA, Sams S, Gleisner A, et al. Re-excision rates after breast conserving surgery following the 2014 SSO-ASTRO guidelines. Am J Surg 2017;214:1104-9.

106. Guidi AJ, Tworek JA, Mais DD, et al. Breast Specimen Processing and Reporting With an Emphasis on Margin Evaluation: A College of American Pathologists Survey of 866 Laboratories. Arch Pathol Lab Med 2018;142:496-506.

107. Schnabel F, Boolbol SK, Gittleman M, et al. A randomized prospective study of lumpectomy margin assessment with use of MarginProbe in patients with nonpalpable breast malignancies. Ann Surg Oncol 2014;21:1589-95.

108. Allweis TM, Kaufman Z, Lelcuk S, et al. A prospective, randomized, controlled, multicenter study of a real-time, intraoperative probe for positive margin detection in breast-conserving surgery. Am J Surg 2008;196:483-9.

109. Kupstas A, Ibrar W, Hayward RD, et al. A novel modality for intraoperative margin assessment and its impact on re-excision rates in breast conserving surgery. Am J Surg 2018;215:400-3.

110. Coble J, Reid V. Achieving clear margins. Directed shaving using MarginProbe, as compared to a full cavity shave approach. Am J Surg 2017;213:627-30.

111. Sebastian M, Akbari S, Anglin B, et al. The impact of use of an intraoperative margin assessment device on reexcision rates. Springerplus 2015;4:198.

112. Blohmer JU, Tanko J, Kueper J, et al. MarginProbe(c) reduces the rate of re-excision following breast conserving surgery for breast cancer. Arch Gynecol Obstet 2016;294:361-7.

113. Dixon JM, Renshaw L, Young O, et al. Intraoperative assessment of excised breast tumour margins using ClearEdge imaging device. Eur J Surg Oncol 2016;42:1834-40.

114. Erickson-Bhatt SJ, Nolan RM, Shemonski ND, et al. Realtime Imaging of the Resection Bed Using a Handheld

Cite this article as: McEvoy MP, Landercasper J, Naik HR, Feldman S. Update of the American Society of Breast Surgeons Toolbox to address the lumpectomy reoperation epidemic. Gland Surg 2018;7(6):536-553. doi: 10.21037/gs.2018.11.03
Probe to Reduce Incidence of Microscopic Positive Margins in Cancer Surgery. Cancer Res 2015;75:3706-12. 115.Zysk AM, Chen K, Gabrielson E, et al. Intraoperative Assessment of Final Margins with a Handheld Optical Imaging Probe During Breast-Conserving Surgery May Reduce the Reoperation Rate: Results of a Multicenter Study. Ann Surg Oncol 2015;22:3356-62.

116. Wang YW, Reder NP, Kang S, et al. RamanEncoded Molecular Imaging with Topically Applied SERS Nanoparticles for Intraoperative Guidance of Lumpectomy. Cancer Res 2017;77:4506-16.

117. Thomas G, Nguyen TQ, Pence IJ, et al. Evaluating feasibility of an automated 3-dimensional scanner using Raman spectroscopy for intraoperative breast margin assessment. Sci Rep 2017;7:13548.

118. Mondal SB, Gao S, Zhu N, et al. Optical See-Through Cancer Vision Goggles Enable Direct Patient Visualization and Real-Time Fluorescence-Guided Oncologic Surgery. Ann Surg Oncol 2017;24:1897-903.

119. Unkart JT, Chen SL, Wapnir IL, et al. Intraoperative Tumor Detection Using a Ratiometric Activatable Fluorescent Peptide: A First-in-Human Phase 1 Study. Ann Surg Oncol 2017;24:3167-73.

120. Klimberg VS, Ochoa D, Henry-Tillman R, et al. Longterm results of phase II ablation after breast lumpectomy added to extend intraoperative margins (ABLATE 1) trial. J Am Coll Surg 2014;218:741-9.

121. Killelea BK, Yang VQ, Mougalian S, et al. Neoadjuvant chemotherapy for breast cancer increases the rate of breast conservation: results from the National Cancer Database. J Am Coll Surg 2015;220:1063-9.

122. Broecker JS, Hart AM, Styblo TM, et al. Neoadjuvant Therapy Combined With Oncoplastic Reduction for High-Stage Breast Cancer Patients. Ann Plast Surg 2017;78:S258-62.

123. Abe SE, Hill JS, Han Y, et al. Margin re-excision and local recurrence in invasive breast cancer: A cost analysis using a decision tree model. J Surg Oncol 2015;112:443-8. 